\title{
MINIMUM ENERGY SOLUTION FOR THE SPHERICAL SHELL
}

\author{
BY \\ R. W. DICKEY \\ University of Wisconsin, Madison
}

\begin{abstract}
A Galerkin procedure is used to prove the existence of a minimum energy solution for the problem of the spherical shell under constant normal pressure. It is shown that if the pressure is sufficiently small the trivial solution is the minimum energy solution and if the pressure is sufficiently large a nontrivial solution furnishes the minimum energy solution. Bounds are obtained on these critical pressures.
\end{abstract}

1. Introduction. In this paper we shall discuss rotationally symmetric solutions of the spherical shell under constant normal pressure. Experimental results indicate (cf. [1]) that as the normal pressure is increased the spherical shell shrinks into a smaller spherical shell until at some critical value of the pressure buckling occurs in the form of a small dimple. This buckling occurs at a pressure significantly lower than that predicted by linear shell theory and the form of the buckled state differs from that predicted by the linear theory.

The spherical shell problem has generated a large literature (cf. the references in [2-4]). Much of this literature is devoted to describing the bifurcation structure, which numerical evidence indicates is quite complicated (cf. [3]), and in obtaining qualitative information on the various nontrivial solutions (cf. $[2,5]$ ).

In this paper we will show that if the applied normal pressure is sufficiently large there exists a nontrivial solution whose energy is less than the trivial solution. Bounds will be determined on the critical pressure at which the bifurcation occurs. It will be shown that the critical pressure is always less than or equal to the lowest eigenvalue of the linear shell theory. In certain cases, depending on the shell thickness, the critical pressure is definitely less than the lowest eigenvalue of the linear shell theory. The method is essentially A Galerkin procedure similar to that used in treating the nonlinear bending of circular plates (cf. [6]).

Equations describing the axisymmetric behavior of a spherical shell under constant normal pressure were derived in [3]. These equations can be reduced to a pair of

Received June 20, 1989.

(C)1990 Brown University 
nonlinear ordinary differential equations

$$
\begin{gathered}
L q+\nu q=-v-\frac{1}{2} v^{2} \cot \theta \\
L v-\nu v=\gamma(-P v+q+q v \cot \theta)
\end{gathered}
$$

where

$$
L=\frac{d^{2}}{d \theta^{2}}+\cot \theta \frac{d}{d \theta}-(\cot \theta)^{2} .
$$

In (1.1) $q$ is a quantity proportional to the radial bending moment and

$$
v=u+\frac{d w}{d \theta}
$$

where $u$ is the displacement in the $\theta$ direction and

$$
w=W(\theta)-W_{0}
$$

where $W$ is the displacement in the negative radial direction and $W_{0}$ is the radial displacement of the unbuckled state

$$
W_{0}=(1-\nu) P
$$

The other quantities given in (1.1) are

$$
P=\frac{p a}{4 E h}, \quad \gamma=\frac{1-\nu^{2}}{k}, \quad k=\frac{1}{3}\left(\frac{h}{a}\right)^{2}
$$

where $p$ is the applied normal pressure, $a$ is the radius of the middle surface of the shell, $2 h$ is the thickness of the shell, $E$ is the Young's modulus, and $\nu$ is the Poisson ratio. The boundary conditions on (1.1) are

$$
q(0)=q(\pi)=v(0)=v(\pi)=0 .
$$

Once $q$ and $v$ are determined from (1.1) and (1.6) other quantities of interest such as radial and circumferential stresses and radial and circumferential displacements can be determined by quadrature (cf. [3]).

2. Reduction of the equations. It is convenient to rewrite Eq. (1.1) in terms of the new independent variable

$$
x=\cos \theta .
$$

The differential operator $L$ (cf. (1.2)) becomes

$$
L=\left(1-x^{2}\right) \frac{d^{2}}{d x^{2}}-2 x \frac{d}{d x}-\frac{x^{2}}{1-x^{2}}
$$

and the two equations (1.1) can be rewritten

$$
\begin{gathered}
(L+\nu) q=-v-\frac{1}{2} v^{2} \frac{x}{\sqrt{1-x^{2}}} \\
(L+\nu) v=2 \nu v-\gamma P v+\gamma q+\gamma q v \frac{x}{\sqrt{1-x^{2}}}
\end{gathered}
$$


with boundary conditions

$$
q(-1)=q(1)=v(-1)=v(1)=0 .
$$

The normalized associated Legendre functions (cf. [7]) are defined by

$$
A_{n}^{m}(x)=\left(1-x^{2}\right)^{m / 2} \frac{d P_{n}(x)}{d x} /\left\|\left(1-x^{2}\right)^{m / 2} P_{n}^{\prime}\right\|
$$

where $P_{n}(x)$ is the Legendre polynomial of order $n$. In particular

$$
A_{n}^{1}=A_{n}=\sqrt{1-x^{2}} P_{n}^{\prime} /\left\|\left(1-x^{2}\right)^{1 / 2} P_{n}^{\prime}\right\|
$$

$\left.{ }^{\prime}=d / d x\right)$. The functions $A_{n}$ are the eigenfunctions of the problem

$$
\begin{gathered}
L A_{n}+(n(n+1)-1) A_{n}=0 \\
A_{n}(-1)=A_{n}(1)=0 .
\end{gathered}
$$

Equation (2.7) may be rewritten in the form

$$
(L+\nu) A_{n}=-\lambda_{n} A_{n}
$$

where

$$
\lambda_{n}=n(n+1)-(1+\nu)
$$

for $n \geq 1$. Equation (2.9) with boundary conditions (2.8) can be rewritten as a Fredholm integral equation

$$
A_{n}=\lambda_{n} G A_{n}
$$

where $G=-(L+\nu)^{-1}$. The operator $G$ is simply the integral operator whose kernel is the Green's function for $-(L+\nu)$ with boundary conditions (2.8), i.e.,

$$
G u=\int_{-1}^{1} g(x, \xi) u(\xi) d \xi
$$

where $g(x, \xi)$ is symmetric and continuous (cf. [8]). Introduce the inner product

$$
\langle u, v\rangle=\int_{-1}^{1} u(x) v(x) d x .
$$

It is an immediate consequence of the above remarks that $G$ is syn..metric, i.e., $\langle G u, v\rangle=\langle u, G v\rangle$, and positive, i.e., $\langle G u, u\rangle>0$ for all $u \neq 0$.

The pair of equations (2.3) can be reduced to a single nonlinear integral equation for the determination of $v$. Using the fact that $(L+\nu)^{-1}=-G$, rewrite Eq. (2.3a) in the form

$$
q=G v+\frac{1}{2} G \frac{x v^{2}}{\sqrt{1-x^{2}}} .
$$

Combining (2.3b) and (2.14) we find

$$
\begin{aligned}
(L+\nu) v= & 2 \nu v-\gamma P v+\gamma G v \\
& +\frac{\gamma}{2} G \frac{x v^{2}}{\sqrt{1-x^{2}}}+\frac{\gamma x v}{\sqrt{1-x^{2}}} G v+\frac{1}{2} \frac{\gamma x v}{\sqrt{1-x^{2}}} G \frac{x v^{2}}{\sqrt{1-x^{2}}} .
\end{aligned}
$$


Rewriting (2.15) as an integral equation yields

$$
\begin{aligned}
v= & -2 \nu G v+\gamma P G v-\gamma G G v-\frac{\gamma}{2} G\left(G \frac{x v^{2}}{\sqrt{1-x^{2}}}\right) \\
& -\gamma G\left(\frac{x v}{\sqrt{1-x^{2}}} G v\right)-\frac{\gamma}{2} G\left(\frac{x v}{\sqrt{1-x^{2}}} G \frac{x v^{2}}{\sqrt{1-x^{2}}}\right) \\
= & F(v) .
\end{aligned}
$$

3. The Galerkin expansion. Assume for the moment that Eq. (2.16) has a solution. If this solution is sufficiently smooth it can be expanded in a series

$$
v=\sum_{n=1}^{\infty} a_{n} A_{n}
$$

This series would satisfy Eq. (2.16), i.e.,

$$
\begin{aligned}
\sum_{n=1}^{\infty} a_{n} A_{n}= & -2 \nu \sum_{n=1}^{\infty} \frac{a_{n}}{\lambda_{n}} A_{n}+\gamma P \sum_{n=1}^{\infty} \frac{a_{n}}{\lambda_{n}} A_{n} \\
& -\gamma \sum_{n=1}^{\infty} \frac{a_{n}}{\lambda_{n}^{2}} A_{n}-\frac{\gamma}{2} G\left(G \frac{x v^{2}}{\sqrt{1-x^{2}}}\right) \\
& -\gamma G\left(\frac{x v}{\sqrt{1-x^{2}}} G v\right)-\frac{\gamma}{2} G\left(\frac{x v}{\sqrt{1-x^{2}}} G \frac{x v^{2}}{\sqrt{1-x^{2}}}\right),
\end{aligned}
$$

where we have used (2.11) and $v$ is given by (3.1). If we multiply (3.2) by one of the eigenfunctions $A_{n}$ and integrate from -1 to 1 we obtain the infinite set of nonlinear algebraic equations for the determination of $a_{n}$

$$
\begin{aligned}
a_{n}= & -2 \nu \frac{a_{n}}{\lambda_{n}}+\gamma P \frac{a_{n}}{\lambda_{n}}-\gamma \frac{a_{n}}{\lambda_{n}^{2}}-\frac{\gamma}{2}\left\langle G\left(G \frac{x v^{2}}{\sqrt{1-x^{2}}}\right), A_{n}\right\rangle \\
& -\gamma\left\langle G\left(\frac{x v}{\sqrt{1-x^{2}}} G v\right), A_{n}\right\rangle \\
& -\frac{\gamma}{2}\left\langle G\left(\frac{x v}{\sqrt{1-x^{2}}} G \frac{x v^{2}}{\sqrt{1-x^{2}}}\right), A_{n}\right\rangle
\end{aligned}
$$

for $n=1,2, \ldots$ Using the symmetry of $G$, Eq. (3.3) can be rewritten

$$
\begin{aligned}
\lambda_{n} a_{n} & +2 \nu a_{n}-\gamma P a_{n}+\gamma \frac{a_{n}}{\lambda_{n}}+\frac{\gamma}{2}\left\langle G \frac{x v^{2}}{\sqrt{1-x^{2}}}, A_{n}\right\rangle \\
& +\gamma\left\langle\frac{x v}{\sqrt{1-x^{2}}} G v, A_{n}\right\rangle+\frac{\gamma}{2}\left\langle\frac{x v}{\sqrt{1-x^{2}}} G \frac{x v^{2}}{\sqrt{1-x^{2}}}, A_{n}\right\rangle=0,
\end{aligned}
$$

$n=1,2, \ldots$. 
4. Approximate solutions. The initial object is to prove that the system of Eq. (3.4) has a nontrivial solution. For this purpose we will consider the truncated system

$$
\begin{aligned}
\lambda_{n} a_{n} & +2 \nu a_{n}-\gamma P a_{n}+\gamma \frac{a_{n}}{\lambda_{n}}+\frac{\gamma}{2}\left\langle G \frac{x v_{N}^{2}}{\sqrt{1-x^{2}}}, A_{n}\right\rangle \\
& +\gamma\left\langle\frac{x v_{N}}{\sqrt{1-x^{2}}} G v_{N}, A_{n}\right\rangle+\frac{\gamma}{2}\left\langle\frac{x v_{N}}{\sqrt{1-x^{2}}} G \frac{x v_{N}^{2}}{\sqrt{1-x^{2}}}, A_{n}\right\rangle=0
\end{aligned}
$$

for $n=1,2, \ldots, N$ and

$$
v_{N}=\sum_{n=1}^{N} a_{n} A_{n} .
$$

The finite system (4.1) always has the trivial solution $a_{1}=\cdots=a_{N}=0$. The object is to show that under some circumstances there is a nontrivial solution.

We introduce a function $E_{N}\left(a_{1}, \ldots, a_{N}\right)$ which is essentially the energy associated with (4.1),

$$
\begin{aligned}
E_{N}= & \sum_{n=1}^{N}\left(\lambda_{n}+2 \nu-\gamma P+\frac{\gamma}{\lambda_{n}}\right) a_{n}^{2} \\
& +\gamma\left\langle G \frac{x v_{N}^{2}}{\sqrt{1-x^{2}}}, v_{N}\right\rangle+\frac{\gamma}{4}\left\langle G \frac{x v_{N}^{2}}{\sqrt{1-x^{2}}}, \frac{x v_{N}^{2}}{\sqrt{1-x^{2}}}\right\rangle .
\end{aligned}
$$

The symmetry of $G$ implies that Eq. (4.1) is

$$
\frac{1}{2} \frac{\partial E_{N}}{\partial a_{n}}=0, \quad n=1,2, \ldots, N .
$$

Thus the solutions of Eq. (4.1) are critical points of $E_{N}$, and conversely. In order to prove the existence of a solution to (4.1) it suffices to show that $E_{N}$ has a minimum. For this purpose it is convenient to rewrite (4.3) in the form

$$
\begin{aligned}
E_{N}= & \sum_{n=1}^{N}\left(\lambda_{n}+2 \nu-\gamma P\right) a_{n}^{2} \\
& +\frac{\gamma}{4}\left\langle G\left(\frac{x v_{N}^{2}}{\sqrt{1-x^{2}}}+2 v_{N}\right), \frac{x v_{N}^{2}}{\sqrt{1-x^{2}}}+2 v_{N}\right\rangle .
\end{aligned}
$$

Since $G$ is a positive operator, (4.5) implies that $E_{N} \geq 0$ if $\lambda_{1}+2 \nu-\gamma P \geq 0$, i.e., if (cf. (2.10))

$$
P \leq \frac{1}{\gamma}(1+\nu)=\frac{1}{3(1-\nu)}\left(\frac{h}{a}\right)^{2} .
$$

If $P$ satisfies the inequality (4.6) the minimum of (4.3) occurs for $a_{1}=\cdots=a_{N}=0$. Thus if $P$ satisfies (4.6) the minimum energy solution is the trivial solution.

The quantities

$$
P=\frac{1}{\gamma}\left(\lambda_{j}+2 \nu\right)+\frac{1}{\lambda_{j}}
$$

are the eigenvalues of the linear shell theory (cf. [3]). Assume $\min _{j} P_{j}$ occurs for $j=$ $n$. In this case $P_{n} \leq P_{n-1}$ and $P_{n} \leq P_{n+1}$. It follows that a necessary and sufficient 
condition for $P_{n}$ to be the smallest eigenvalue is that the thickness parameter $\gamma$ satisfy

$$
\lambda_{n} \lambda_{n-1} \leq \gamma \leq \lambda_{n} \lambda_{n+1}
$$

We shall show that if $P>P_{n}$ ( $n$ odd) or $P \geq P_{n}$ ( $n$ even) there are values $\left(a_{1}, \ldots, a_{N}\right)$ such that $E_{N}\left(a_{1}, \ldots, a_{N}\right)<0$. In particular

$$
\begin{aligned}
E_{N}\left(0, \ldots, a_{n}, \ldots, 0\right)= & \left(\lambda_{n}+2 \nu-\gamma P+\frac{\gamma}{\lambda_{n}}\right) a_{n}^{2} a_{n}^{3} \gamma\left\langle G \frac{x A_{n}^{2}}{\sqrt{1-x^{2}}}, A_{n}\right\rangle \\
& +\frac{a_{n}^{4}}{4}\left\langle G \frac{x A_{n}^{2}}{\sqrt{1-x^{2}}}, \frac{x A_{n}^{2}}{\sqrt{1-x^{2}}}\right\rangle
\end{aligned}
$$

and (cf. (2.6))

$$
\left\langle G \frac{x A_{n}^{2}}{\sqrt{1-x^{2}}}, A_{n}\right\rangle=\frac{1}{\lambda_{1}}\left\langle\frac{x A_{n}^{2}}{\sqrt{1-x^{2}}}, A_{n}\right\rangle=\frac{1}{\lambda_{n}} \int_{-1}^{1} x\left(1-x^{2}\right)\left(P_{n}^{\prime}\right)^{3} d x .
$$

If $n$ is odd the integral in (4.8) vanishes since the integrand is odd. If $n$ is even the integral in (4.8) does not vanish (in fact it is positive, cf. [10]). In this case $E_{N}\left(0, \ldots, a_{n}, \ldots, 0\right)<0$ even in the case $\lambda_{n}+2 \nu-\gamma P+\gamma / \lambda_{n}=0$ for the appropriate choice of $a_{n}$. Thus a sufficient condition to guarantee the minimum of $E_{N}\left(a_{1}, \ldots, a_{N}\right)$ is nonzero is

$$
P \leq P_{n}=\frac{1}{\gamma}\left(\lambda_{n}+2 \nu\right)+\frac{1}{\lambda_{n}} .
$$

We will prove the existence of a solution to (4.1) by proving that $E_{N}\left(a_{1}, \ldots, a_{N}\right)$ has a minimum. Given this result it is a consequence of (4.6) and (4.10) that there is some value $P=P_{0}$

$$
\frac{1}{\gamma}(1+\nu)<P_{0} \leq \frac{1}{\gamma}\left(\lambda_{n}+2 \nu\right)+\frac{1}{\lambda_{n}}
$$

(where the inequality becomes a strict inequality if $n$ is even) such that if $P>P_{0}$ the minimum of $E_{N}$ is negative, i.e., the solution of (4.1) which minimizes the energy is nonzero.

There is no difficulty in showing that $E_{N} \rightarrow \infty$ as $\left(a_{1}^{2}+\cdots+a_{N}^{2}\right)^{1 / 2} \rightarrow \infty$ (cf. (4.3)). We would also like to show that $E_{N}$ has a lower bound which is independent of $N$. An immediate consequence is that $E_{N}$ has a minimum. This minimum furnishes a nontrivial solution of (4.1) if $P>P_{0}$.

In order to find a lower bound on $E_{N}$ it is useful to rewrite (4.3) in the form

$$
\begin{aligned}
E_{N}= & \sum_{n=1}^{N}\left(\lambda_{n}+2 \nu-\gamma P+\frac{\gamma}{\lambda_{n}}\right) a_{n}^{2}+\frac{\gamma}{8}\left\langle G \frac{x v_{N}^{2}}{\sqrt{1-x^{2}}}, \frac{x v_{N}^{2}}{\sqrt{1-x^{2}}}\right\rangle \\
& +\frac{\gamma}{8}\left\langle G\left(\frac{x v_{N}^{2}}{\sqrt{1-x^{2}}}+4 v_{N}\right), \frac{x v_{N}^{2}}{\sqrt{1-x^{2}}}+4 v_{N}\right\rangle-2 \gamma\left\langle G v_{N}, v_{N}\right\rangle .
\end{aligned}
$$


Equation (4.12) can be simplified by noting that

$$
\left\langle G v_{N}, v_{N}\right\rangle=\sum_{n=1}^{N} \frac{a_{n}^{2}}{\lambda_{n}}
$$

so that

$$
\begin{aligned}
E_{N}= & \sum_{n=1}^{N}\left(\lambda_{n}+2 \nu-\gamma P-\frac{\gamma}{\lambda_{n}}\right) a_{n}^{2} \\
& +\frac{\gamma}{8}\left\langle G\left(\frac{x v_{N}^{2}}{\sqrt{1-x^{2}}}+4 v_{N}\right), \frac{x v_{N}^{2}}{\sqrt{1-x^{2}}}+4 v_{N}\right\rangle \\
& +\frac{\gamma}{8}\left\langle G \frac{x v_{N}^{2}}{\sqrt{1-x^{2}}}, \frac{x v_{N}^{2}}{\sqrt{1-x^{2}}}\right\rangle .
\end{aligned}
$$

Since $\lambda_{n} \geq \lambda_{1}=1-\nu$, Eq. (4.14) can be replaced by the inequality

$$
\begin{aligned}
E_{N} \geq & \sum_{n=1}^{N}\left(\lambda_{n}+2 \nu-\gamma P-\frac{\gamma}{1-\nu}\right) a_{n}^{2} \\
& +\frac{\gamma}{8}\left\langle G\left(\frac{x v_{N}^{2}}{\sqrt{1-x^{2}}}+4 v_{N}\right), \frac{x v_{N}^{2}}{\sqrt{1-x^{2}}}+4 v_{N}\right\rangle \\
& +\frac{\gamma}{8}\left\langle G \frac{x v_{N}^{2}}{\sqrt{1-x^{2}}}, \frac{x v_{N}^{2}}{\sqrt{1-x^{2}}}\right\rangle .
\end{aligned}
$$

In order to simplify the notation define

$$
\begin{aligned}
F_{N} & =\sum_{n=1}^{N}\left(\lambda_{n}+2 \nu-\gamma P-\frac{\gamma}{1-\nu}\right) a_{n}^{2} \\
& =\int_{-1}^{1}\left(v_{N} \sum_{n=1}^{N} a_{n} \lambda_{n} A_{n}\right) d x+\left(2 \nu-\gamma P-\frac{\gamma}{1-\nu}\right) \int_{-1}^{1} v_{N}^{2} d x
\end{aligned}
$$

or, using (2.9),

$$
F_{N}=-\int_{-1}^{1} v_{N}(L+\nu) v_{N} d x+\left(2 \nu-\gamma P-\frac{\gamma}{1-\nu}\right) \int_{-1}^{1} v_{N}^{2} d x .
$$

After an integration by parts (4.17) can be rewritten

$$
F_{N}=\int_{-1}^{1}\left\{\left(1-x^{2}\right)\left(v_{N}^{\prime}\right)^{2}+\frac{x^{2}}{1-x^{2}} v_{N}^{2}+\nu v_{N}^{2}-\left(\gamma P+\frac{\gamma}{1-\nu}\right) v_{n}^{2}\right\} d x .
$$

Thus (4.15) can be written

$$
\begin{aligned}
E_{n} \geq & F_{n}+\frac{\gamma}{8}\left\langle G\left(\frac{x v_{N}^{2}}{\sqrt{1-x^{2}}}+4 v_{N}\right), \frac{x v_{N}^{2}}{\sqrt{1-x^{2}}}+4 v_{N}\right\rangle \\
& +\frac{\gamma}{8}\left\langle G \frac{x v_{N}^{2}}{\sqrt{1-x^{2}}}, \frac{x v_{N}^{2}}{\sqrt{1-x^{2}}}\right\rangle .
\end{aligned}
$$


In order to obtain our lower bound on $E_{N}$ we note that if $g(x)$ is any continuous function on $-1 \leq x \leq 1$ we have

$$
\begin{aligned}
\left\langle G \frac{x v_{N}^{2}}{\sqrt{1-x^{2}}}, \frac{x v_{N}^{2}}{\sqrt{1-x^{2}}}\right\rangle= & \left\langle G\left(\frac{x v_{N}^{2}}{\sqrt{1-x^{2}}}-g\right), \frac{x v_{N}^{2}}{\sqrt{1-x^{2}}}-g\right\rangle \\
& +2\left\langle\frac{x v_{N}^{2}}{\sqrt{1-x^{2}}}, G g\right\rangle-\langle G g, g\rangle .
\end{aligned}
$$

Combining (4.19) and (4.20) we find

$$
\begin{aligned}
E_{N} \geq & H_{N}+\frac{\gamma}{8}\left\langle G\left(\frac{x v_{N}^{2}}{\sqrt{1-x^{2}}}+4 v_{N}\right), \frac{x v_{N}^{2}}{\sqrt{1-x^{2}}}+4 v_{N}\right\rangle \\
& +\frac{\gamma}{8}\left\langle G\left(\frac{x v_{N}^{2}}{\sqrt{1-x^{2}}}-g\right), \frac{x v_{N}^{2}}{\sqrt{1-x^{2}}}-g\right\rangle-\frac{\gamma}{8}\langle G g, g\rangle
\end{aligned}
$$

where

$$
H_{N}=F_{N}+\frac{\gamma}{4}\left\langle\frac{x v_{N}^{2}}{\sqrt{1-x^{2}}}, G g\right\rangle .
$$

The object is to choose $g(x)$ in such a way as to guarantee that $H_{N} \geq 0$ for all $N$. If this is possible for some fixed $g$ it will follow that (cf. (4.21)) $E_{N} \geq-\gamma\langle G g, g\rangle / 8$, i.e., we would have a lower bound on $E_{N}$ which is independent of $N$. For this purpose we note that

$$
\begin{aligned}
& \min _{a_{1}, \ldots, a_{N}} H_{N} \\
& \quad \geq \min _{v \neq 0} H \\
& \quad=\min _{v \neq 0} \int_{-1}^{1}\left\{\left(1-x^{2}\right)\left(v^{\prime}\right)^{2}+\left(\frac{x^{2}}{1-x^{2}}+\nu-\left(\gamma P+\frac{\gamma}{1-\nu}\right)+\frac{\gamma x G g}{4 \sqrt{1-x^{2}}}\right) v^{2}\right\} d x
\end{aligned}
$$

where the minimum on the right side of (4.23) is taken over all $v \in C_{2}[-1,1]$ satisfying $v(-1)=v(1)=0$. The variational characterization of eigenvalues (cf. [8]) guarantees that

$$
H \geq \mu \int_{-1}^{1} v^{2} d x
$$

where $\mu$ is the smallest eigenvalue of the linear Sturm-Liouville problem

$$
\left(\left(1-x^{2}\right) v^{\prime}\right)^{\prime}+\left(\mu-\frac{x^{2}}{1-x^{2}}-\nu+\gamma P+\frac{\gamma}{1-\nu}-\frac{\gamma x G g}{4 \sqrt{1-x^{2}}}\right) v=0
$$

with boundary conditions

$$
v(-1)=v(1)=0
$$

The smallest eigenvalue of

$$
\left(\left(1-x^{2}\right) v^{\prime}\right)^{\prime}+\left(\mu-\frac{x^{2}}{1-x^{2}}-\nu\right) v=0
$$


with boundary conditions (4.26) is $\mu=1+\nu>0$ and the corresponding eigenfunctions is $v=A_{1}(x)=\left(1-x^{2}\right)^{1 / 2}$. If we could choose $g(x)$ so as to reduce (4.25) to (4.26) it would follow that $H_{N} \geq 0$. However, this is not possible since

$$
\frac{x G g}{4 \sqrt{1-x^{2}}}=f(x)
$$

vanishes at $x=0$. Instead we will choose $f(x)$ so that Eqs. (4.25) and (4.26) agree over most of the interval $-1 \leq x \leq 1$. In particular for small $\varepsilon>0$ define $f(x)$ by

$$
f(x)=\left(P+\frac{1}{1-\nu}\right) h(x)
$$

where

$$
h(x)= \begin{cases}1, & \varepsilon \leq|x| \leq 1 \\ 3\left(\frac{x}{\varepsilon}\right)^{2}-3\left(\frac{x}{\varepsilon}\right)^{4}+\left(\frac{x}{\varepsilon}\right)^{6}, & |x| \leq \varepsilon .\end{cases}
$$

It is easily verified that $f(x) \in C_{2}[-1,1]$ and $f(0)=0$. We must verify that there exists a continuous function $g(x)$ such that $(4.28)$ is satisfied, i.e, we must show that

$$
g(x)=-(L+\nu) \frac{4 \sqrt{1-x^{2}}}{x} f(x)
$$

is continuous for $-1 \leq x \leq 1$.

If $\varepsilon \leq|x| \leq 1$ Eqs. (4.29) and (4.31) imply that

$$
g(x)=-4\left(P+\frac{1}{1-\nu}\right)(L+\nu) \frac{\sqrt{1-x^{2}}}{x} .
$$

The right side of (4.32) may be calculated to show

$$
g(x)=-4\left(P+\frac{1}{1-\nu}\right)\left(\frac{\left(x^{2}+2\right) \sqrt{1-x^{2}}}{x^{3}}+\frac{\nu \sqrt{1-x^{2}}}{x}\right)
$$

when $\varepsilon \leq|x| \leq 1$. Thus $g(x)$ is continuous for $\varepsilon \leq|x| \leq 1$. If $|x| \leq \varepsilon$ Eqs. (4.29) and (4.31) imply that

$$
g(x)=-4\left(P+\frac{1}{1-\nu}\right)(L+\nu) \sqrt{1-x^{2}}\left\{\frac{3 x}{\varepsilon^{2}}-\frac{3 x^{2}}{\varepsilon^{4}}+\frac{x^{5}}{\varepsilon^{6}}\right\} .
$$

There is no difficulty in showing from (4.34) that $g(x)$ is continuous for $|x| \leq \varepsilon$. In addition $g(x)$ is continuous at $x= \pm \varepsilon$ since $f(x)$ is twice continuous differentiable over the whole interval.

It remains to show that when $\varepsilon$ is sufficiently small the smallest eigenvalue of (4.25) and (4.26) is close to the smallest eigenvalue $\mu=1+\nu$ of the problem (4.27) and (4.26). It would follow that the smallest eigenvalue of (4.25) and (4.26) is positive when $\varepsilon$ is sufficiently small. It is possible to calculate the smallest eigenvalue of (4.25) and (4.26) by perturbation. The actual calculation is carried out in the Appendix (Sec. 6). In any case the result is

$$
\mu=1+\nu+\frac{\gamma}{c}\left(P+\frac{1}{1-\nu}\right) \varepsilon+O\left(\varepsilon^{2}\right)
$$

where $c$ is a positive constant. 
In view of the above remarks the inequality (4.21) implies that when $\varepsilon$ is sufficiently small

$$
M(N)=\min _{a_{1}, \ldots, a_{N}} E_{N}\left(a_{1}, \ldots, a_{N}\right) \geq-\frac{\gamma}{8}\langle G g, g\rangle
$$

where $g(x)$ is given by (4.33) when $\varepsilon \leq|x| \leq 1$ and (4.34) when $|x| \leq \varepsilon$. The bound on $M(N)$ is independent of $N$. It is also possible to obtain an upper bound on $E_{N}$ which is independent of $N$. In particular

$$
\begin{aligned}
M(N) & =\min _{a_{1}, \ldots, a_{N}} E_{N}\left(a_{1}, \ldots, a_{N}\right) \leq \min _{a_{1}} E_{N}\left(a_{1}, 0, \ldots, 0\right) \\
& =\min _{a_{1}} E_{1}\left(a_{1}\right)=M(1) .
\end{aligned}
$$

We have already noted that if $P>P_{0}$ (cf. (4.11)) is satisfied then $M(1)<0$.

5. The exact solution. In Sec. 4 we have shown that the finite system (4.1) has a nontrivial solution $a_{1}(N), \ldots, a_{N}(N)$ if $P>P_{0}$. We wish to use this fact to prove the existence of a solution to the infinite system (3.4). The solution of the finite system satisfies the inequalities

$$
-\frac{\gamma}{8}\langle G g, g\rangle \leq E_{N}\left(a_{1}(N), \ldots, a_{N}(N)\right) \leq M(1)
$$

where $M(1)<0$ if $P>P_{0}$. In addition $a_{1}(N), \ldots, a_{N}(N)$ satisfies the identity

$$
\begin{gathered}
\sum_{n=1}^{N}\left(\lambda_{n}+2 \nu-\gamma P+\frac{\gamma}{\lambda_{n}}\right) a_{n}^{2}(N)+\frac{3 \gamma}{2}\left\langle G \frac{x v_{N}^{2}(N)}{\sqrt{1-x^{2}}}, v_{N}(N)\right\rangle \\
+\frac{\gamma}{2}\left\langle G \frac{x v_{N}^{2}(N)}{\sqrt{1-x^{2}}}, \frac{x v_{N}^{2}(N)}{\sqrt{1-x^{2}}}\right\rangle=0
\end{gathered}
$$

where

$$
v_{N}(N)=\sum_{n=1}^{N} a_{n}(N) A_{n}(x) .
$$

The identity (5.2) is obtained by multiplying (4.1) by $a_{n}$ and summing over $n$.

The identity (5.2) can be rewritten

$$
\begin{aligned}
\sum_{n=1}^{N} & \left(\lambda_{n}+2 \nu-\gamma P+\frac{\gamma}{\lambda_{n}}\right) a_{n}^{2}(N) \\
& +\frac{\gamma}{2}\left\langle G\left(\frac{x v_{N}^{2}(N)}{\sqrt{1-x^{2}}}+3 v_{N}(N)\right), \frac{x v_{N}^{2}(N)}{\sqrt{1-x^{2}}}+3 v_{N}(N)\right\rangle \\
& -\frac{9 \gamma}{8}\left\langle G v_{N}(N), v_{N}(N)\right\rangle=0
\end{aligned}
$$

or using (4.13) and the positivity of $G$

$$
\sum_{n=1}^{N}\left(\lambda_{n}+2 \nu-\gamma P-\frac{\gamma}{8 \lambda_{n}}\right) a_{n}^{2}(N) \leq 0 .
$$


It is a consequence of $(5.5)$ that

$$
\sum_{n=1}^{N} n^{2} a_{n}^{2}(N) \leq \gamma\left(P+\frac{1}{8(1-\nu)}\right) \sum_{n=1}^{N} a_{n}^{2}(N) .
$$

It remains to bound the right side of (5.6). This bound comes from (5.1). It follows from (4.24) that

$$
H_{N}\left(a_{1}(N), \ldots, a_{N}(N)\right) \geq \mu \int_{-1}^{1} v_{N}^{2}(N) d x
$$

where $\mu>0$. Thus $(4.21)$ implies

$$
\begin{aligned}
E_{N} & \geq \mu \int_{-1}^{1} v_{N}^{2}(N) d x-\frac{\gamma}{8}\langle G g, g\rangle \\
& =\mu \sum_{n=1}^{N} a_{N}^{2}(N)-\frac{\gamma}{8}\langle G g, g\rangle .
\end{aligned}
$$

Combining (5.8) and (5.1) we find

$$
\sum_{n=1}^{N} a_{n}^{2}(N) \leq \frac{1}{\mu}\left(M(1)+\frac{\gamma}{8}\langle G g, g\rangle\right) .
$$

The inequalities (5.6) and (5.9) imply the existence of constants $K_{1}$ and $K_{2}$, independent of $N$, such that

$$
\sum_{n=1}^{N} a_{n}^{2}(N) \leq K_{1}, \quad \sum_{n=1}^{N} n^{2} a_{n}^{2}(N) \leq K_{2} .
$$

Define a vector

$$
V_{N}=\left(a_{1}(N), \ldots, a_{N}(N), 0, \ldots\right) .
$$

We wish to show that the sequence $\left\{V_{N}\right\}$ has a convergent subsequence. For this purpose let $\ell_{2}$ be the infinite dimensional real vector space with elements $x=$ $\left(x_{1}, x_{2}, \ldots, x_{n}, \ldots\right)$ and norm

$$
\|x\|=\left(\sum_{n=1}^{\infty} x_{n}^{2}\right)^{1 / 2}
$$

In addition we consider a subset of $\ell_{2}$

$$
s=\left\{x \in \ell_{2} \mid \sum_{n=1}^{\infty} n^{2} x_{n}^{2} \leq K_{2}\right\} .
$$

Let $x \in s$ and let $x^{*}=\left(x_{1}, \ldots, x_{n}, 0, \ldots\right)$. The fact that $x \in s$ implies that $x_{j}^{2} \leq K_{2} / j^{2}$ from which it follows that

$$
\left\|x-x^{*}\right\|^{2}=\sum_{j=n+1}^{\infty} x_{j}^{2} \leq K_{2} \sum_{j=n+1}^{\infty} \frac{1}{j^{2}} .
$$


The right side of (5.13) can be made arbitrarily small by choosing $n$ sufficieritly large. This result proves that $s$ is a compact subset of $\ell_{2}$ (cf. [9]). The sequence $\left\{V_{N}\right\} \subset s$. Since $s$ is compact there exists a subsequence $\left\{V_{N_{i}}\right\}$ such that

$$
V_{N_{i}} \rightarrow V^{*}=\left(a_{1}^{*}, a_{2}^{*}, \ldots, a_{n}^{*}, \ldots\right),
$$

and $V^{*} \in s$, i.e.,

$$
\sum_{n=1}^{\infty} n^{2}\left(a_{n}^{*}\right)^{2} \leq K_{2}
$$

Define the function

$$
v^{*}=\sum_{n=1}^{\infty} a_{n}^{*} A_{n}(x)
$$

The function $v^{*}(x)$ is continuous and the sequence of functions $v_{N_{i}}\left(N_{i}\right) \rightarrow v^{*}$ uniformly. The proof of these two facts is essentially identical to the proof given in [6]. The components $a_{n}^{*}$ of the vector $V^{*}$ given in (5.14) are a solution of the infinite system of algebraic equation (3.4). In order to show this we use the fact that $a_{n}\left(N_{i}\right)$ is a solution of the finite system (4.1) to write

$$
\begin{aligned}
\mid\left(\lambda_{n}+2 \nu\right. & \left.-\gamma P+\frac{\gamma}{\lambda_{n}}\right) a_{n}^{*}+\frac{\gamma}{2}\left\langle G \frac{x\left(v^{*}\right)^{2}}{\sqrt{1-x^{2}}}, A_{n}\right\rangle \\
+\gamma & \left\langle\frac{x v^{*}}{\sqrt{1-x^{2}}} G v^{*}, A_{n}\right\rangle+\frac{\gamma}{2}\left\langle\frac{x v^{*}}{\sqrt{1-x^{2}}} G \frac{x\left(v^{*}\right)^{2}}{\sqrt{1-x^{2}}}, A_{n}\right\rangle \mid \\
\leq & \left|\left(\lambda_{n}+2 \nu-\gamma P+\frac{\gamma}{\lambda_{n}}\right)\left(a_{n}^{*}-a_{n}\left(N_{i}\right)\right)\right| \\
& +\frac{\gamma}{2}\left|\left\langle G \frac{x\left(v^{*}\right)^{2}}{\sqrt{1-x^{2}}}, A_{n}\right\rangle-\left\langle G \frac{x v_{N_{i}}^{2}\left(N_{i}\right)}{\sqrt{1-x^{2}}}, A_{n}\right\rangle\right| \\
& +\gamma\left|\left\langle\frac{x v^{*}}{\sqrt{1-x^{2}}} G v^{*}, A_{n}\right\rangle-\left\langle\frac{x v_{N_{i}}\left(N_{i}\right)}{\sqrt{1-x^{2}}} G v_{N_{i}}\left(N_{i}\right), A_{n}\right\rangle\right| \\
+ & \frac{\gamma}{2}\left|\left\langle\frac{x v^{*}}{\sqrt{1-x^{2}}} G \frac{x\left(v^{*}\right)^{2}}{\sqrt{1-x^{2}}}, A_{n}\right\rangle-\left\langle\frac{x v_{N_{i}}\left(N_{i}\right)}{\sqrt{1-x^{2}}} G \frac{x v_{N_{i}}^{2}\left(N_{i}\right)}{\sqrt{1-x^{2}}}, A_{n}\right\rangle\right| .
\end{aligned}
$$

The right side of (5.17) tends to zero as $N_{i} \rightarrow \infty$. Thus $a_{n}^{*}$ satisfies (3.4).

In order to prove that $v^{*}$, defined in $(5.16)$, is a solution of $(2.16)$ we note that

$$
v^{*}-F\left(v^{*}\right)=h(x)
$$

where $h(x)$ is a continuous function. However,

$$
\left\langle h, A_{n}\right\rangle=\left\langle v^{*}-F\left(v^{*}\right), A_{n}\right\rangle=0, \quad n=1,2, \ldots
$$

since $V^{*}$ is a solution of (3.4). The associated Legendre functions $A_{n}$ form a complete set of eigenfunctions. Thus we conclude that $h(x) \equiv 0$, i.e., $v^{*}$ satisfies (2.16). Recall that $E_{N} \leq M(1)<0$ for all $N$ so that the solution is not zero. 
6. Appendix. In this section we will compute the smallest eigenvalue of (4.25) with boundary conditions (4.26). It is convenient to rewrite (4.25) in the form

$$
\left(\left(1-x^{2}\right) v^{\prime}\right)^{\prime}+\left(\mu-\nu+1-\frac{1}{1-x^{2}}+\gamma P+\frac{\gamma}{1-\nu}-\frac{x G g}{4 \sqrt{1-x^{2}}}\right) v=0 .
$$

We will compare this equation to the equation

$$
\left(\left(1-x^{2}\right) v^{\prime}\right)^{\prime}+\left(\mu-\nu+1-\frac{1}{1-x^{2}}\right) v=0 .
$$

The solution of (6.1) and (6.2) is to satisfy the boundary condition (4.26). The smallest eigenvalue $\mu$ of $(6.2)$ satisfies

$$
\mu-\nu+1=2
$$

and the corresponding eigenfunction is $v=A_{1}(x)=\left(1-x^{2}\right)^{1 / 2}$.

We want to describe a perturbation scheme which will allow us to compute the smallest eigenvalue of (6.1) in terms of $\varepsilon$ (cf. (4.30)). We begin by rewriting (6.1) in the form

$$
\left(\left(1-x^{2}\right) v^{\prime}\right)^{\prime}+\left(2-\delta-\frac{1}{1-x^{2}}+\gamma\left(P+\frac{1}{1-\nu}\right)(1-h(x))\right) v=0 .
$$

We will compute $\delta$ as a series in $\varepsilon$, or equivalently the smallest eigenvalue $\mu=$ $1-\nu-\delta$ as a series in $\varepsilon$.

There is no loss of generality if we replace the boundary conditions $(4.26)$ by the condition

$$
v(-1)=v^{\prime}(0)=0 .
$$

If (6.4) and (6.5) are satisfied on the interval $-1 \leq x \leq 0$, the solution on $0 \leq x \leq 1$ is easily obtained by reflection. We begin by solving (6.4) on the interval $-\varepsilon \leq x \leq 0$ with boundary conditions

$$
v(-\varepsilon)=1, \quad v^{\prime}(0)=0 .
$$

Introduce the change of variable

$$
\xi=\frac{x}{\varepsilon} .
$$

Equation (6.4) with boundary conditions $(6.6)$ becomes

$$
\begin{aligned}
& \frac{d}{d \xi}\left[\left(1-\varepsilon^{2} \xi^{2}\right) \frac{d v}{d \xi}\right] \\
& +\left[(2-\delta) \varepsilon^{2}=\frac{\varepsilon^{2}}{1-\varepsilon^{2} \xi^{2}}+\varepsilon^{2} \gamma\left(P+\frac{1}{1-\nu}\right)\left(1-3 \xi^{2}+3 \xi^{4}-\xi^{6}\right)\right] v=0 \\
& v(-1)=1,\left.\quad \frac{d v}{d \xi}\right|_{\xi=0}=0 .
\end{aligned}
$$

The problem (6.8) and (6.9) will have a solution of the form

$$
v(\xi)=v_{0}(\xi)+\varepsilon^{2} v_{1}(\xi)+\cdots
$$


where $v_{0}$ is a solution of the problem

$$
\begin{gathered}
\frac{d^{2}}{d \xi^{2}} v_{0}=0 \\
v_{0}(-1)=1,\left.\quad \frac{d}{d \xi} v_{0}\right|_{\xi=0}=0
\end{gathered}
$$

and

$$
\begin{gathered}
\frac{d^{2}}{d \xi^{2}} v_{1}=-\left(1-\delta+\gamma\left(P+\frac{1}{1-\nu}\right)\right) v_{0} \\
v_{1}(-1)=0,\left.\quad \frac{d}{d \xi} v_{1}\right|_{\xi=0}=0 .
\end{gathered}
$$

This pair of problems is easily solved to find

$$
v_{0}=1, \quad v_{1}=\frac{1}{2}\left(1-\delta+\gamma\left(P+\frac{1}{1-\nu}\right)\right)\left(1-\xi^{2}\right)
$$

so that

$$
v=1+\frac{\varepsilon^{2}}{2}\left(1-\delta+\gamma\left(P+\frac{1}{1-\nu}\right)\right)\left(1-\left(\frac{x}{\varepsilon}\right)^{2}\right)+\cdots
$$

for $-\varepsilon \leq x \leq 0$.

It remains to solve the problem on the interval $-1 \leq x \leq-\varepsilon$. On this interval Eq. (6.4) is

$$
\left(\left(1-x^{2}\right) v^{\prime}\right)^{\prime}+\left(1-\delta-\frac{1}{1-x^{2}}\right) v \equiv 0
$$

and we will apply the boundary conditions

$$
v(-1)=0, \quad v(-\varepsilon)=1 .
$$

The problem (6.17) and (6.18) has a solution which may be written in the form

$$
v=v_{0}+\delta v_{1}+\cdots
$$

where

$$
\begin{aligned}
& \left(\left(1-x^{2}\right) v_{0}^{\prime}\right)^{\prime}+\left(2-\frac{1}{1-x^{2}}\right) v_{0}=0 \\
& \left(\left(1-x^{2}\right) v_{1}^{\prime}\right)^{\prime}+\left(2-\frac{1}{1-x^{2}}\right) v_{1}=v_{0} .
\end{aligned}
$$

The boundary conditions are

$$
\begin{array}{ll}
v_{0}(-1)=0, & v_{0}(-\varepsilon)=1 \\
v_{1}(-1)=0, & v_{1}(-\varepsilon)=0 .
\end{array}
$$

The solution of (6.20) satisfying the conditions $(6.22)$ is

$$
v_{0}=\frac{\sqrt{1-x^{2}}}{\sqrt{1-\varepsilon^{2}}} .
$$


The solution of $(6.21)$ is technically more difficult. It can be solved by variation of parameters, but first we need the general solution of the homogeneous equation. One solution of $(6.20)$ is

$$
u_{1}=\sqrt{1-x^{2}}
$$

In order to determine another independent solution let $v=u_{2}=u_{1} w$. Placing this in $(6.28)$ we fine

$$
w^{\prime \prime}-\frac{4 x}{1-x^{2}} w^{\prime}=0 .
$$

This equation is easily solved to show

$$
w^{\prime}=\frac{c}{\left(1-x^{2}\right)^{2}}
$$

where $c$ is an arbitrary constant. After integrating (6.27) and multiplying the result by $u_{1}$ we have a second independent solution of the homogeneous problem

$$
u_{2}=\frac{x}{\sqrt{1-x^{2}}}+\frac{1}{2} \sqrt{1-x^{2}} \ln \left(\frac{1+x}{1-x}\right)
$$

A particular solution of the inhomogeneous problem (6.21) is of the form $v=c_{1} u_{1}+$ $c_{2} u_{2}$ where $c_{1}$ and $c_{2}$ must satisfy

$$
\begin{gathered}
c_{1}^{\prime} u_{1}+c_{2}^{\prime} u_{2}=0 \\
c_{1}^{\prime} u_{1}^{\prime}+c_{2}^{\prime} u_{2}^{\prime}=\frac{2}{\sqrt{1-\varepsilon^{2}} \sqrt{1-x^{2}}} .
\end{gathered}
$$

$c_{1}^{\prime}$ and $c_{2}^{\prime}$ are determined from (6.29) to be

$$
\begin{gathered}
c_{1}^{\prime}=\frac{2 x+\left(1-x^{2}\right) \ln \left(\frac{1+x}{1-x}\right)}{\sqrt{1-\varepsilon^{2}}\left(3+x^{2}\right)} \\
c_{2}^{\prime}=\frac{2\left(1-x^{2}\right)}{\sqrt{1-\varepsilon^{2}}\left(3+x^{2}\right)}
\end{gathered}
$$

so that

$$
\begin{gathered}
c_{1}=\frac{1}{\sqrt{1-\varepsilon^{2}}} \int_{-1}^{x} \frac{2 \xi+\left(1-\xi^{2}\right) \ln \left(\frac{1+\xi}{1-\xi}\right)}{\left(3+\xi^{2}\right)} d \xi \\
c_{2}=\frac{2}{\sqrt{1-\varepsilon^{2}}} \int_{-1}^{x} \frac{1-\xi^{2}}{3+\xi^{2}} d \xi .
\end{gathered}
$$


The solution of (6.21) satisfying (6.23) is

$$
\begin{aligned}
v_{1}= & -\frac{\sqrt{1-x^{2}}}{\sqrt{1-\varepsilon^{2}}} \int_{-1}^{x} \frac{2 \xi+\left(1-\xi^{2}\right) \ln \left(\frac{1+\xi}{1-\xi}\right)}{3+\xi^{2}} d \xi \\
& +\frac{2}{\sqrt{1-\varepsilon^{2}}}\left(\frac{x}{\sqrt{1-x^{2}}}+\frac{1}{2} \sqrt{1-x^{2}} \ln \left(\frac{1+x}{1-x}\right)\right) \int_{-1}^{x} \frac{1-\xi^{2}}{3+\xi^{2}} d \xi \\
+ & \sqrt{1-x^{2}}\left\{\frac{1}{\sqrt{1-\varepsilon^{2}}} \int_{-1}^{-\varepsilon} \frac{2 \xi+\left(1-\xi^{2}\right) \ln \left(\frac{1+\xi}{1-\xi}\right)}{3+\xi^{2}} d \xi\right. \\
& \left.\quad-\frac{2}{\sqrt{1-\varepsilon^{2}}}\left(-\frac{\varepsilon}{1-\varepsilon^{2}}+\frac{1}{2} \ln \left(\frac{1+\varepsilon}{1-\varepsilon}\right)\right) \int_{-1}^{-\varepsilon} \frac{1-\xi^{2}}{3+\xi^{2}} d \xi\right\} .
\end{aligned}
$$

For our purposes it is only necessary to retain terms of sufficiently small order in $\varepsilon$ and $\delta$. In particular (cf. (6.24))

$$
\begin{gathered}
v_{0}=\sqrt{1-x^{2}}+O\left(\varepsilon^{2}\right) \\
v_{1}=-\sqrt{1-x^{2}} \int_{-1}^{x} \frac{2 \xi+\left(1-\xi^{2}\right) \ln \left(\frac{1+\xi}{1-\xi}\right)}{3+\xi^{2}} d \xi \\
+\left(\frac{x}{\sqrt{1-x^{2}}}+\frac{1}{2} \sqrt{1-x^{2}} \ln \left(\frac{1+x}{1-x}\right)\right) \int_{-1}^{x} \frac{1-\xi^{2}}{3+\xi^{2}} d \xi \\
+\sqrt{1-x^{3} 2} \int_{-1}^{0} \frac{2 \xi+\left(1-\xi^{2}\right) \ln \left(\frac{1+\xi}{1-\xi}\right)}{3+\xi^{2}} d \xi+O(\varepsilon) .
\end{gathered}
$$

In the interval $-1 \leq x \leq-\varepsilon$ the solution of (6.4) and (6.5) is given by (6.19) where $v_{0}$ and $v_{1}$ are given by (6.33) and (6.34). In the interval $-\varepsilon \leq x \leq 0$ the solution of (6.4) and (6.5) is given by (6.16). There is one more requirement. We need to determine $\delta$ so that $v(x)$ has two continuous derivatives at $x=-\varepsilon$. We already know that $v(x)$ is continuous at $x=-\varepsilon$. Thus we determine $\delta$ so that $v^{\prime}(x)$ is continuous at $x=-\varepsilon$. The continuity of the second derivative follows from the differential equation.

Equation (6.16) implies that

$$
\begin{aligned}
\lim _{x \downarrow-\varepsilon} v^{\prime}(x) & =\varepsilon\left(1-\delta+\gamma\left(P+\frac{1}{1-\nu}\right)\right)+\cdots \\
& =\varepsilon\left(1+\gamma\left(P+\frac{1}{1-\nu}\right)\right)+\cdots
\end{aligned}
$$

where the remainder in (6.35) consists of a term which is quadratic in the two parameters $\varepsilon$ and $\delta$. Turning to $(6.33)$ and $(6.34)$ we find

$$
v_{0}^{\prime}=-\frac{x}{\sqrt{1-x^{2}}}+O\left(\varepsilon^{2}\right)
$$




$$
\begin{aligned}
v_{1}^{\prime}(x)= & \frac{x}{\sqrt{1-x^{2}}} \int_{-1}^{x} \frac{2 \xi+\left(1+\xi^{2}\right) \ln \left(\frac{1+\xi}{1-\xi}\right)}{3+\xi^{2}} d \xi \\
& -\sqrt{1-x^{2}}\left(\frac{2 x+\left(1-x^{2}\right) \ln \left(\frac{1+x}{1-x}\right)}{3+x^{2}}\right) \\
& +2\left(\frac{1}{\left(1-x^{2}\right)^{3 / 2}}+\frac{1}{2} \frac{1}{\sqrt{1-x^{2}}}-\frac{1}{2} \frac{x}{\sqrt{1-x^{2}}} \ln \left(\frac{1+x}{1-x}\right)\right) \int_{-1}^{x} \frac{1-\xi^{2}}{3+\xi^{2}} d \xi \\
& +2\left(\frac{x}{\sqrt{1-x^{2}}}+\frac{1}{2} \sqrt{\left.1-x^{2} \ln \left(\frac{1+x}{1-x}\right)\right) \frac{1-x^{2}}{3+x^{2}}}\right. \\
& -\frac{x}{\sqrt{1-x^{2}}} \int_{-1}^{0} \frac{2 \xi+\left(1-\xi^{2}\right) \ln \left(\frac{1+\xi}{1-\xi}\right)}{3+\xi^{2}} d \xi
\end{aligned}
$$

There is no difficulty in showing that

$$
\begin{gathered}
v_{0}^{\prime}(-\varepsilon)=\varepsilon+O\left(\varepsilon^{2}\right) \\
v_{1}^{\prime}(-\varepsilon)=3 \int_{-1}^{0} \frac{1-\xi^{2}}{3+\xi^{2}} d \xi+O(\varepsilon) .
\end{gathered}
$$

Combining (6.37) and (6.38) with (6.19) we find

$$
\lim _{x \uparrow-\varepsilon} v^{\prime}(x)=\varepsilon+3 \delta \int_{-1}^{0} \frac{1-\xi^{2}}{3+\xi^{2}} d \xi+\cdots
$$

where the remainder is quadratic in the two parameters $\varepsilon$ and $\delta$. Comparing (6.35) and (6.39) we find that continuity of $v^{\prime}(x)$ at $x=-\varepsilon$ requires

$$
\varepsilon+3 \delta \int_{-1}^{0} \frac{1-\xi^{2}}{3+\xi^{2}} d \xi=\varepsilon\left(1+\gamma\left(P+\frac{1}{1-\nu}\right)\right)+\cdots
$$

Equation (4.35) follows from (6.40).

Added in proof. In showing that the constructed function $v^{*}$ is a solution of the integral equation (2.16) it was indicated that the proof is identical to that given in [6]. This proof requires that the eigenfunction be bounded independent of $n$. Actually the functions $A_{n}$ grow with $n$ and therefore a modification is required.

The function

$$
H(x, \xi)=g(x, \xi)-\sum_{j=1}^{N} \frac{1}{\lambda_{j}} A_{j}(x) A_{j}(\xi)
$$

generates a nonnegative operator, i.e., $\langle H U, U\rangle \geq 0$. It follows that

$$
\sum_{j=1}^{\infty} \frac{1}{\lambda_{j}} A_{j}(x)^{2} \leq g(x, x) \leq M
$$

(cf. [8, p. 38]). This result in conjunction with (5.10) and (5.15) is sufficient to guarantee that $v_{N_{i}} \rightarrow v^{*}$ pointwise as $N_{i} \rightarrow \infty$. It also follows that $v^{*}$ is bounded 
since

$$
\left|v^{*}\right| \leq\left(\sum_{j=1}^{\infty} \lambda_{j}\left(a_{j}^{*}\right)^{2} \sum_{j=1}^{\infty} \frac{1}{\lambda_{j}} A_{j}^{2}\right)^{1 / 2}
$$

The sums on the right of (3) are bounded (cf. (2) and (5.15)).

We wish to show that $a_{n}^{*}$ is a solution of (3.4). Since $a_{n}\left(N_{i}\right)$ is a solution of (4.1) we take the limit as $N_{i} \rightarrow \infty$ to find

$$
\begin{aligned}
\left(\lambda_{n}+2 v+v P\right. & \left.+\frac{\gamma}{\lambda_{n}}\right) a_{n}^{*} \\
& +\lim _{N_{i} \rightarrow \infty} \gamma\left\{\frac{1}{2}\left\langle G \frac{x v_{N_{i}}^{2}}{\sqrt{1-x^{2}}}, A_{n}\right\rangle+\left\langle\frac{x v_{N_{i}}}{\sqrt{1-x^{2}}} G v_{N_{i}}, A_{n}\right\rangle\right. \\
& \left.+\frac{1}{2}\left\langle\frac{x v_{N_{i}}}{\sqrt{1-x^{2}}} G \frac{x v_{N_{i}}^{2}}{\sqrt{1-x^{2}}}, A_{n}\right\rangle\right\}=0 .
\end{aligned}
$$

It remains to show that the limit can be taken inside the integrals in (4). The argument in each case is similar. Since $v_{N_{i}} \rightarrow v^{*}$

$$
\lim _{N_{i} \rightarrow \infty} g(x, \xi) \frac{\xi v_{N_{i}}^{2}}{\sqrt{1-\xi^{2}}}=g(x, \xi) \frac{\xi\left(v^{*}\right)^{2}}{\sqrt{1-\xi^{2}}}
$$

when $\xi \neq \pm 1$. In addition

$$
\left|g(x, \xi) \frac{\xi v_{N_{i}}^{2}}{\sqrt{1-\xi^{2}}}\right| \leq C \frac{|\xi g(x, \xi)|}{\sqrt{1-\xi^{2}}}
$$

where $C$ is a bound on $\left(v^{*}\right)^{2}$. The right side of $(6)$ is integrable so that the dominated convergence theorem implies

$$
\lim _{N_{i} \rightarrow \infty} \int_{-1}^{1} g(x, \xi) \frac{\xi v_{N_{i}}^{2}}{\sqrt{1-\xi^{2}}} d \xi=\int_{-1}^{1} g(x, \xi) \frac{\xi\left(v^{*}\right)^{2}}{\sqrt{1-\xi^{2}}} d \xi .
$$

The same argument shows that

$$
\lim _{N_{i} \rightarrow \infty}\left\langle\frac{x v_{N_{i}}}{\sqrt{1-x^{2}}} G \frac{x v_{N_{i}}^{2}}{\sqrt{1-x^{2}}}, A_{n}\right\rangle=\left\langle\frac{x v^{*}}{\sqrt{1-x^{2}}} G \frac{x\left(v^{*}\right)^{2}}{\sqrt{1-x^{2}}}, A_{n}\right\rangle .
$$

A similar argument can be used to show that the limit can be taken inside the integral in each of the terms occurring in (4). Thus $a_{n}^{*}$ is a solution of (3.4) and it is a consequence that $(\mathrm{cf} .(2.16))$

$$
\left\langle v^{*}-F\left(v^{*}\right), A_{n}\right\rangle=0, \quad n=1,2, \ldots
$$

Thus $v^{*}$ is a solution of the integral equation (2.16). 


\section{REFERENCES}

[1] J. J. Stoker, Nonlinear Elasticity, Gordon and Breach, New York, 1968

[2] S. S. Antman, Existence and nonuniqueness of axisymmetric equilibrium states of nonlinearly elastic shells, Arch. Rational Mech. Anal 40, 329-373 (1981)

[3] L. Bauer, E. L. Reiss, and H. B. Keller, Axisymmetric buckling of hollow spheres and hemispheres, Comm. Pure and Appl. Math 23, 529-568 (1970)

[4] C. G. Lange and G. A. Kriegsmann, The axisymmetric branching behavior of complete spherical shells, Quart. Appl. Math. 39, 145-178 (1981)

[5] K. G. Shih and S. S. Antman, Qualitative properties of large buckled states of spherical shells, Arch. Rational Mech. Anal. 93, 357-384 (1986)

[6] R. W. Dickey, Nonlinear bending of circular plates, SIAM J. Appl. Math. 30, 1-9 (1976)

[7] N. N. Lebedev, Special Functions and Their Applications, Prentice-Hall, Englewood Cliffs, N.J., 1965

[8] R. Courant and D. Hilbert, Methods of Mathematical Physics, Vol 1., Interscience, New York, 1953

[9] A. N. Kolmogorov and S. V. Fomin, Elements of the Theory of Functions and Functional Analysis, Vol 1., Graylock Press, Rochester, N.Y., 1957

[10] R. A. Askey, Orthogonal polynomials and special function, Regional Conference Series in Applied Math, Vol. 21., SIAM, Philadelphia 